\title{
Anne Applebaum, 2019, Famine rouge, Grasset, Paris, 512 pages
}

\author{
Iryna DMYTRYCHYN
}

Le livre d'Anne Applebaum, journaliste et historienne, spécialiste de l'Europe de l'Est dont les travaux sur le Goulag ont été couronnés par le prestigieux Prix Pulitzer, s'attèle à l'étude d'un des aspects du stalinisme, la Grande famine en Ukraine 1932-1933, appelée le Holodomor, de la contraction de deux racines, holod [famine, faim] et moryty [exténuer, tuer].

Près de quarante ans après l'œuvre pionnière de Robert Conquest (The Harvest of Sorrow - Les Sanglantes Moissons, 1986), c'est la première grande enquête sur la famine ukrainienne dans l'historiographie occidentale, même si d'éminents historiens lui ont consacré des études importantes dans le cadre de leurs travaux sur la collectivisation en URSS dont on citera en premier lieu en France Nicolas Werth.

Famine rouge s'ouvre sur les révolutions ukrainiennes à la suite de la chute des empires russe et austro-hongrois, et s'achève sur les événements actuels, le Maïdan (2013-2014), l'annexion de la Crimée (2014) et la guerre dans l'Est de l'Ukraine (2014-). Cet élargissement de cadre permet de placer le Holodomor dans une perspective longue, en en expliquant la genèse et en en éclairant les conséquences jusqu'à nos jours. La plongée dans les événements des années 1917-1920 et, en particulier, la conquête bolchévique de l'Ukraine, outre le fait d'apporter des informations factuelles, expose les raisons qui ont conditionnées la politique de Staline à l'égard de l'Ukraine. Car l'auteure est persuadée que celle-ci trouve son explication dans son expérience de ces années révolutionnaires, lorsqu'il était commissaire aux nationalités. Redoutant le soulèvement ukrainien, Staline s'est appliqué à mater la campagne avec la plus grande fermeté, prenant la lutte de survie de cette dernière comme une manifestation de l'irrédentisme ukrainien. Le 
SLOVO

Les Voyages lointains des écrivains polonais $\left(\mathrm{XX}^{\mathrm{e}}-\mathrm{XXI}^{\mathrm{e}}\right.$ siècles $)-\mathrm{n}^{\circ} 51$

sous-titre du livre - « La guerre de Staline contre l'Ukraine » - n'est pas anodin : le Holodomor ne relève pas du domaine agricole, mais du politique et n'est pas une question d'action irréfléchie, mais celle d'une logique, aussi inconcevable soit-elle.

La famine elle-même - le cœur du récit - se dévoile au travers d'une chronique implacable, réunissant les rapports internes, la correspondance des responsables de tous les niveaux, y compris du plus haut échelon de l'État, les témoignages des survivants et des observateurs, pour aboutir à une trame complexe d'une grande précision. La mise en chapitres chronologique et thématique très détaillée permet de couvrir tous les aspects, dès les prémisses de la collectivisation aux conséquences de la famine à court et moyen termes, mais aussi son camouflage et sa présence dans la mémoire. L'auteure aborde également les aspects concomitants à la famine, indispensables pour saisir l'ensemble du tableau, à savoir la destruction des élites ukrainiennes : politiques, culturelles, religieuses.

Sorti en 2017, le livre a provoqué de nombreuses réactions parmi les historiens, comme en témoignent des interventions aux quatre coins de la planète. En Ukraine, la discussion la plus acharnée tournait autour de la qualification de la famine en tant que génocide, soulignant la haute sensibilité de la question. À l'évidence, beaucoup s'attendaient à ce qu'Anne Applebaum réponde par la positive et son absence d'engagement a été mal reçue. Or, il s'agit là d'un procès d'intention: certes, Anne Applebaum n'a pas dit que le Holodomor était un génocide. Mais elle n'a pas dit non plus que ce n'en était pas un. Elle a simplement dit que le Holodomor n'entre pas dans la définition du génocide telle que stipulé par la Convention pour la prévention et la répression du crime de génocide de 1948, mais relèverait de sa définition élargie. Si elle insiste qu'il n'appartient pas à un historien de se prononcer sur les questions de qualification juridique, Anne Applebaum invite, par son exposé des faits, tout un chacun à forger sa propre conviction.

Anne Appelbaum étaye de manière convaincante la façon dont la famine peut donner des éléments d'explications non seulement de l'Ukraine actuelle, mais aussi de la Russie, pour qui la « perte » de l'Ukraine (dans la version actuelle, l'arrimage de l'Ukraine à l'Europe), semble être toujours difficile à admettre ou est synonyme d'une menace, s' inscrivant dans ce qu'elle appelle, « les schémas anciens ».

Si l'auteure s'est attachée à montrer l'impact de la famine sur le développement de l'Ukraine, avec ses effets négatifs qui se font toujours sentir, elle conclut cependant avec une note positive, sur le fait que Staline qui, par peur de perdre l'Ukraine, a détruit ses élites, attaqué sa langue et sa culture, décimé sa paysannerie, in fine a échoué : l'Ukraine existe. Les stigmates du Holodomor non seulement n'empêchent pas d'avancer, mais aujourd'hui d'autres épisodes, plus récents, fondent la nation, laissant le passé entre les mains des historiens. 
Réunissant une somme impressionnante de documents d'archives, de témoignages, s'appuyant sur les travaux des dernières décennies, Famine rouge est servi par une analyse aussi rigoureuse que fine et profonde, et s'impose d'emblée comme un livre de référence pour comprendre un des plus grands crimes de la période stalinienne. 